\title{
Pulmonary Agenesis with Dextrocardia and Hypertrophic Cardiomyopathy: First Case Report
}

Sheetal Agarwal, Arti Maria*, Dinesh Yadav and Narendra Bagri

Department of Pediatrics, Ram Manohar Lohia Hospital, New Delhi, India

*Corresponding author: Arti Maria, Dept. of Pediatrics, Ram Manohar Lohia Hospital, New Delhi, India, Tel: +919818618586; E-mail: artimaria@gmail.com

Rec date: April 17, 2014, 2014; Acc date: May 23, 2014; Pub date: May 25, 2014

Copyright: @ 2014 Agarwal S, et al. This is an open-access article distributed under the terms of the Creative Commons Attribution License, which permits unrestricted use, distribution, and reproduction in any medium, provided the original author and source are credited.

\begin{abstract}
Pulmonary agenesis is a rare condition with complete absence of bronchus, lung tissue and vessels. A variety of cardiovascular defects are present in upto $1 / 3 \mathrm{rd}$ cases of pulmonary agenesis. However, a combination of dextrocardia and hypertrophic cardiomyopathy in association with pulmonary agenesis is not known. Here we report the first case of a neonate presenting with respiratory distress since birth, diagnosed to have hypertrophic cardiomyopathy in association with dextrocardia, multiple cardiac defects and right lung agenesis. Association of heart disease with lung agenesis adversely affects the course and outcome making them a highly lethal association.
\end{abstract}

Keywords: Pulmonary agenesis; Dextrocardia; Hypertrophic cardiomyopathy; Neonate

\section{Introduction}

Pulmonary agenesis is a rare condition with reported prevalence of 3.4/1 lakh live births [1]. A variety of cardiovascular defects present in upto $1 / 3$ rd cases of pulmonary agenesis are an important determinant of prognosis [2]. So far an association of pulmonary agenesis with dextrocardia and Hypertrophic Cardiomyopathy (HCM) has not been reported. Here we report such a neonate symptomatic since birth.

\section{Case Summary}

A 22 day old male baby was brought with breathing difficulty since birth. Baby was born with birth weight of $2.4 \mathrm{~kg}$ at term to an elderly non diabetic mother out of a non- consanguineous marriage. Dextrocardia had been diagnosed prenatally on level II ultrasonogram. Baby presented with heart rate $-160 / \mathrm{min}$, respiratory rate- $78 / \mathrm{min}$, subcostal, intercostal retractions and cyanosis (SPO2-70\%) which improved with oxygen. Perfusion was normal. Brachial pulses were better palpable than femorals. Right upper limb systolic blood pressure was $24 \mathrm{mmHg}$ higher than in right lower limb. Baby had low set ears, low hair line, a high arched palate, undescended right testis and hypospadias. Chest examination revealed absent air entry on right side with hyperresonance on left side. Cardiac exam revealed apex beat palpable in the right 4 th intercostal space and a grade $3 / 6$ systolic murmur at the right sternal border. There was no hepatosplenomegaly. X-Ray chest showed homogenous white out on right side with crowding of ribs and no air bronchogram. Mediastinal shift to the right was noted. There was hyperinflation of left lung with pneumonic patch in the upper zone. Electrocardiogram was unremarkable. 2-D Echocardiography revealed dextrocardia with left sided hypoplastic aortic arch (ascending aorta- $6.2 \mathrm{~mm}$, tranverse arch- $3.5 \mathrm{~mm}$, descending aorta- $6.5 \mathrm{~mm}$, proximal transverse arch : diameter of ascending aorta $=56 \%$, distal transverse arch : diameter of ascending aorta $=45 \%$, ) and coarctation of aorta (PG-32 mmHg) with normal aortic valve. There was marked thickening of interventricular septum and concentric hypertrophy of both the ventricles compromising cavity size without obstruction of left or right ventricular outflow tracts. In diastole, the ventricular septum measured $12 \mathrm{~mm}$ in thickness (normal for age -1.5 to $5.5 \mathrm{~mm}$ ) and the posterior wall of left ventricle measured $13 \mathrm{~mm}$ (normal for age 1.5 to $5.5 \mathrm{~mm}$ ). These findings were consistent with diagnosis of hypertrophic cardiomyopathy (HCM) (Figure 1). Septal defects with left to right flow across the shunts were also noted [(ostium secundum atrial septal defect $(3.5 \mathrm{~mm})$, large outlet ventricular septal defect $(6$ $\mathrm{mm})$ and patent ductus srteriosus $(3 \mathrm{~mm})]$. CT angiography revealed complete agenesis of right lung with non visualisation of right pulmonary artery and vein and hypoplastic aortic arch with aberrant retro-esophageal course of right subclavian artery (Figure 2). Infantogram, ultrasound abdomen and cranium excluded other organ anomalies. Screening echo of parents and elder sibling was normal. The family history was negative for HCM or sudden death. A normal tandem Mass Spectroscopy excluded metabolic causes of HCM

Baby was managed with oxygen, intravenous fluids, antibiotics, decongestive therapy and beta blockers. Baby continued to be oxygen dependant with mild respiratory distress and was sent on home oxygen therapy to be kept under regular follow up. HCM was non regressive with the ventricular septal and posterior ventricular wall dimensions remaining same in serial echocardiograms at 1,2 and 3 months.

\section{Discussion}

Pulmonary agenesis is characterised by complete absence of bronchus, lung tissue and vessels. Normally the lung develops as a diverticulm from foregut in the 4 th week of intrauterine life. Pulmonary agenesis results due to failure of the bronchial analogue to divide equally between the two lung buds leading to normal development of one side while failure of other side to develop either completely (agenesis/aplasia) or partially (dysplasia or hypoplasia) [3]. Since the migration of the heart to left side occurs at this period of intrauterine life, developmental anomalies of lung and malposition of heart commonly co-exist [4]. Genetic, teratogenic and mechanical factors have been implicated in the etiology of pulmonary agenesis $[5,6]$. Karyotype was normal in the index case. However no etiological 
diagnosis could be confirmed as genetic mutation analysis was not affordable.

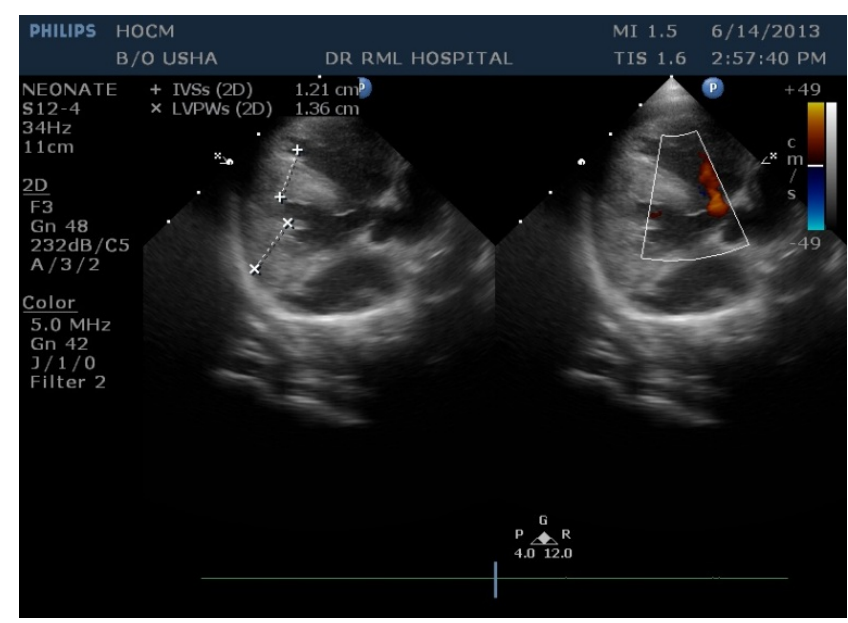

Figure 1: 2-D Echo showing septal hypertrophy (IVSs-1.21 cm) and ventricular wall hypertrophy (LVPWs-1.36 cm) with arrow pointing VSD

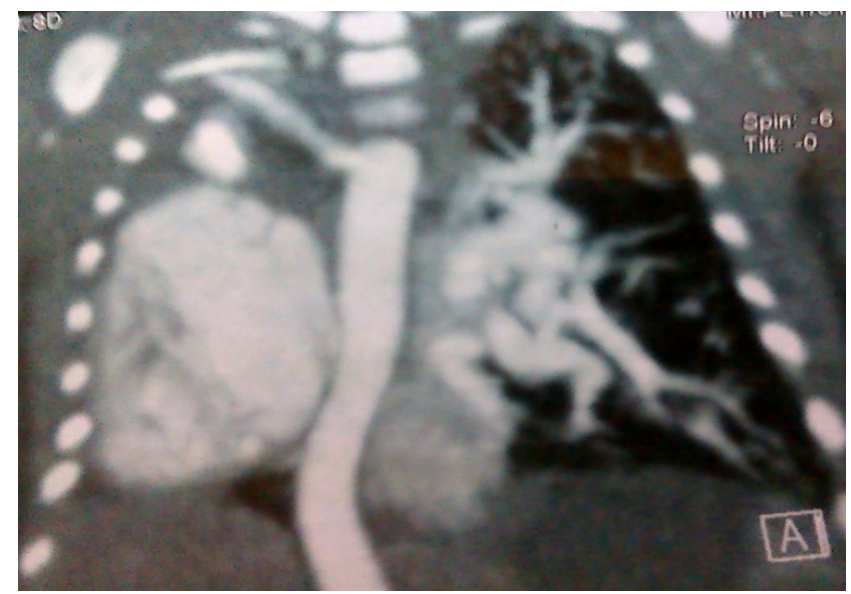

Figure 2: CT Angiograph showing complete agenesis of right lung with non-visualisation of right pulmonary artery and vein with arrow pointing to hypoplastic aortic arch

While bilateral pulmonary agenesis is incompatible with life, $50 \%$ of those with even unilateral lung agenesis die within 5 years of life [7]. Pulmonary agenesis may affect right or left lung equally [3]. The clinical course of individuals with pulmonary agenesis is highly variable; may remain completely asymptomatic till adulthood or present with respiratory insufficiency since birth. The predominant symptoms in newborn period pertain to respiratory tract and include cough, tachypnea, recurrent respiratory infections and cyanosis [4]. Associated anomalies (cardiovascular, skeletal, genitourinary and gastrointestinal) present in upto $50 \%$ of the cases may modify clinical presentation, course and outcome of patients with pulmonary agenesis [8]. Right pulmonary agenesis is more likely than left to be associated with severe symptoms and poor prognosis as it is commonly associated with cardiovascular anomalies, a greater magnitude of shift of heart and a distortion in the course of the great vessels and trachea $[4,9]$.

Associated cardiac anomalies reported so far include ASD, PDA, VSD, Tetralogy of Fallot (TOF), Co-A, right aortic arch, truncus arteriosus and rarely interrupted aortic arch $[2,10]$.

So far, less than 10 cases of dextrocardia with normal heart have been reported in association with right pulmonary agenesis [11]. Cardiomyopathy in association with lung agenesis has only been reported in a 2 year old child so far. However, a combination of dextrocardia and HCM in association with pulmonary agenesis has never been reported.

HCM typically presents as an isolated cardiac lesion. Transient HCM in infancy has been described as a result of exposure to maternal metabolic disorders or corticosteroids. HCM may also be associated with genetic syndromes and rarely as a primary lesion with other heart defects. Clinical course and prognosis of primary HCM in infancy is generally poor.

HCM in our case presented in early neonatal period. Baby continued to be oxygen dependant with compensated $\mathrm{CHF}$ at 3 months. HCM was still non -regressive at 3 months of follow up. HCM in this baby is likely to be primary as other causes had been reasonably well excluded Degree of co-arctation could not explain severe cardiomyopathy in our case. Genetic mutation analysis was not done as it could not be afforded.

This case posed a unique therapeutic challenge as respiratory distress was compounded by pulmonary insufficiency due to agenesis of lung and congestive cardiac failure due to intracardiac shunts and HCM. Left to right cardiac shunts are likely to predispose to repeated chest infections further compromising the respiratory reserve in a baby with pulmonary agenesis.

To the best of our knowledge, HCM with dextrocardia in association with pulmonary agenesis has not been reported in a neonate.

Serious underlying heart disease must be considered if a patient with lung agenesis presents in neonatal period. Association of heart disease with lung agenesis adversely affects the course and outcome. In conclusion, combined anomalies of pulmonary agenesis and congenital heart disease make them a highly lethal association.

\section{References}

1. Mardini MK, Nyhan WL (1985) Agenesis of the lung. Report of four patients with unusual anomalies. Chest 87: 522-527.

2. Meschan I (1987) Radiology of the diaphram, pleura, thoracic cage and upper air passages: Roentgen signs in diagnostic imaging. (2ndedtn), WB Saunders Co, Philadelphia, London, UK.

3. Skandalakis JE, GraySW, Symbas P (1994) The trachea and the lungs: Embryology for Surgeons. (2ndedtn) Williams and Wilkins, Baltimore, MD,USA.

4. Döhlemann C, Mantel K, Schneider K, Güntner M, Kreuzer E, et al. (1990) Deviated trachea in hypoplasia and aplasia of the right lung: airway obstruction and its release by aortopexy. J Pediatr Surg 25: 290-293.

5. Say B, Carpenter NJ, Giacoia G, Jegathesan S (1980) Agenesis of the lung associated with a chromosome abnormality $(46, \mathrm{XX}, 2 \mathrm{p}+)$. J Med Genet 17: 477-478.

6. Roque AS, Burton EM, Boedy RF, Falls G, Bhatia JS (1997) Unilateral pulmonary agenesis without mediastinal displacement. South Med J 90: 335-337. 
Citation: Agarwal A, Maria A, Yadav D, N Bagri (2014) Pulmonary Agenesis with Dextrocardia and Hypertrophic Cardiomyopathy: First Case Report. J Neonatal Biol 3: 141. doi:10.4172/2167-0897.1000141

Page 3 of 3

7. Krivchenya DU, Rudenko EO, Lysak SV, Dubrovin AG, Khursin VN, et al. (2007) Lung aplasia: anatomy, history, diagnosis and surgical management. Eur J Pediatr Surg 17: 244-250.

8. Razaque MA, Singh S, Singh T (1980) Pulmonary agenesis. Indian J Chest Dis Allied Sci 22: 174-178.

9. Ueda T, Nozoe M, Nakamoto Y, Irie Y, Mizushige K (2011) Right pulmonary agenesis in an elderly woman complicated by transient ischemic attack. Intern Med 50: 463-466.
10. Jonathan D, Peter H (2007) Congenital disorders of the lung: Nelson text book of pediatric. (18thedtn), W.B. Saunders Co, Philadelphia, USA.

11. Kullmann F1, Koch R, Feichtinger W, Giesen H, Schmid M, et al. (1993) [Holt-Oram syndrome in combination with reciprocal translocation, lung hypoplasia and cardiomyopathy]. Klin Padiatr 205: 185-189. 